\section{Comments on the Onsager Reciprocal Relations in the Frictional Formalism of Non-equilibrium Thermodynamics}

\section{HANS VINK}

Institute of Physical Chemistry, University of Uppsala, P.O.Box 532, S-751-21 Uppsala, Sweden

The frictional formalism ${ }^{1-5}$ of non-equilibrium thermodynamics provides a theoretical basis for various transport processes in solution and porous media. ${ }^{6-9} \mathrm{~A}$ main feature of the theory is the Onsager reciprocity law, postulating the symmetry of the matrix of the friction coefficients $\left(f_{\mathrm{ij}}\right)$. The symmetry relations are usually introduced on a postulatory basis. Onsager's original proof of the relations ${ }^{10,11}$ is not applicable to continuous media, since the fluxes are not in general derivable from the thermodynamic state variables. ${ }^{12}$ Although the relations imply that the frictional forced between any two components are equal and opposite in direction, and thus comply to Newton's third law, the argument suffers from the weakness that the forces in question have been pre-averaged and thus are not concerned with forces between individual particles. In the present note it is shown that the reciprocal relations are directly related to the conditions of mechanical equilibrium and to the degrees of freedom of the system.

The relations are proved for isothermal diffusion in a solution of $n$ independent components, although the same arguments apply to transport processes in porous media. In diffusion the following balance-of-force equation holds for the $i$ :th component

$\sum_{j=1}^{n} f_{i j} c_{j}\left(u_{i}-u_{j}\right)=X_{i}$

where $f_{i j}(i, j=1, . . n)$ are the friction coefficients between the components specified by the indexes, $u_{i}$ is the average velocity, $c_{i}$ is the concentration and $X_{i}$ is the generalized force. The latter is of the form

$X_{i}=-v_{i} \operatorname{grad} p-\operatorname{grad} \mu_{i}+F_{i}$

where $v_{i}$ is the partial molar volume, $\mu_{i}$ is the isobaric chemical potential, $F_{i}$ the external force per mole of component $i$ and $p$ is the hydrostatic pressure.
Multiplying eqns. (1) and (2) by $c_{i}$ and summing over all components we obtain

$\sum_{i, j} f_{i j} c_{i} c_{j}\left(u_{i}-u_{j}\right)=-\sum_{i} c_{i} v_{i} \operatorname{grad} p$

$-\sum_{i} c_{i} \operatorname{grad} \mu_{i}+\sum_{i} c_{i} F_{i}$

By definition

$\sum_{i} c_{i} v_{i}=1$

and

$J_{i}=c_{i} u_{i}$

where $J_{i}$ is the flux of component $i$. Also, according to Gibbs-Duhem equation

$\sum_{i} c_{i} \operatorname{grad} \mu_{i}=0$

Inserting these relations into eqn. (3) we obtain

$\sum_{i, j} f_{i j} c_{j} J_{i}-\sum_{i, j} f_{i j} c_{i} J_{j}=\sum_{i} c_{i} F_{i}-\operatorname{grad} p$

The condition for mechanical equiblibium is

$\sum_{i} c_{i} F_{i}-\operatorname{grad} p=0$

Inserting this into eqn. (7) and rearranging the left side (by changing the dummy indexes of the second sum) we have at mechanical equilibrium

$\sum_{i, j}\left(f_{i j}-f_{j i}\right) c_{j} J_{i}=0$

Obviously mechanical equilibrium is a necessary condition for the reciprocal relations to hold.

To show that it is also a sufficient condition we make the substitution

$F_{i j}=f_{i j}-f_{j i}$

and rewrite eqn. (9) in the form

$\sum_{i=1}^{n}\left(\sum_{j=1}^{n} F_{i j} c_{j}\right) J_{i}=0$

For nonvanishing coefficients this equation constitutes a permanent constraint on the diffusive fluxes $J_{i}$ (note that here the condition of mechanical equilibrium is essential; if the right side of 
eqn. (11) does not vanish it is a function of the external forces and the equation is merely another version of the phenomenological equations). However, the only admissible constraint in diffusion is concerned with the volume flux, which normally vanishes in the laboratory-fixed coordinate system

$J_{v}=\sum_{i=1}^{n} v_{i} J_{i}=0$

The two constraints cannot be equal, however, as they transform differently when the coordinate system is changed. Transforming to a coordinate system moving with the constant velocity $-u$ in the original laboratory-fixed coordinate system, we obtain the new fluxes

$J_{i}^{\prime}=c_{i}\left(u+u_{i}\right)=J_{i}+u c_{i}$

Thus, eqn. (12) transforms to

$J_{v}^{\prime}=\sum_{i=1}^{n} v_{i} J_{i}^{\prime}=u \sum_{i=1}^{n} c_{i} v_{i}=u$

On the other hand eqn. (11), being defined in terms of relative velocities only, remains unchanged in the transformation

$\sum_{i=1}^{n}\left(\sum_{j=1}^{n} F_{i j} c_{j}\right) J_{i}^{\prime}=u \sum_{i, j} F_{i j} c_{i} c_{j}=0$

Therefore, the constraint represented by eqn. (11) must vanish, which implies that the coefficients in eqn. (11) all vanish

$\sum_{j=1}^{n} F_{i j} c_{j}=0 ; i=1, \ldots n$

However, eqn. (4) represents the only possible constraint on the concentrations. As eqn. (16) is homogeneous, whereas eqn. (4) is inhomogeneous, they cannot represent the same constraint [putting the first $n-1$ concentrations equal to zero yields $c_{n}=1 / v_{n}$ according to eqn. (4) and $c_{n}=0$ according to eqn. (16)]. Thus, the constraints represented by eqn. (16) must vanish, which implies $F_{i j} \equiv 0$, or

$f_{i j}=f_{j i} ; i, j=1, \ldots n$

The present proof is purely phenomenological and demonstrates that the Onsager reciprocal relations are necessary for the conservation of the degrees of freedom of the system. The condition of mechanical equilibrium is here equivalent to the requirement that the fluxes and forces should be derived from the expression for entropy production, since only at mechanical equilibrium is all work dissipated in the system. Thus, at mechanical equilibrium

$\sum_{i=1}^{n} X_{i} J_{i}=\phi=T \sigma$

where $\phi$ is the dissipation function and $\sigma$ the entropy source strength. The dissipation function can be expressed in a compact form in terms of the relative velocities. From eqns. (1), (5), and (18) we obtain (by changing the dummy indexes and using the symmetry of $f_{i j}$ )

$\phi=\sum_{i, j} f_{i j} c_{i} c_{j}\left(u_{i}^{2}-u_{i} u_{j}\right)=$

$\frac{1}{2}\left[\sum_{i, j} f_{i j} c_{i} c_{j}\left(u_{i}^{2}-u_{i} u_{j}\right)\right.$

$\left.+\sum_{i, j} f_{i j} c_{i} c_{j}\left(u_{j}^{2}-u_{i} u_{j}\right)\right]=$

$\frac{1}{2} \sum_{i, j} f_{i j} c_{i} c_{j}\left(u_{i}-u_{j}\right)^{2}$

This equation demonstrates the positive definite character of the dissipation function. It also shows that the friction coefficients are completely determined in terms of the relative velocities of the components. In the frictional formalism only the coefficients $f_{i i}$ are arbitrary, as they make no contributions to eqn. (1). We may therefore define $f_{i i}=0(i=1, \ldots n)$. Note that with this convention the matrix of the friction coefficients is in general non-singular, which is easily verified when the number of components is small.

1. Lamm, O. Ark. Kemi Mineral. Geol. A 18 (1944) No. 2.

2. Lamm, O. Adv. Chem. Phys. 6 (1964) 291.

3. Klemm, A. Z. Naturforsch. Teil A 8 (1953) 397.

4. Laity, R. W. J. Phys. Chem. 63 (1959) 80.

5. Spiegler, K. S. Trans. Faraday Soc. 54 (1958) 1409.

6. Vink, H. J. Chem. Soc. Faraday Trans. 1, 78 (1982) 3115.

7. Vink, H. J. Chem. Soc. Faraday Trans. 1, 79 (1983) 2355.

8. Vink, H. J. Chem. Soc. Faraday Trans. 1, 79 (1983) 2359.

9. Vink, H. J. Chem. Soc. Faraday Trans. 1. 80 (1984) 507.

10. Onsager, L. Phys. Rev. 37 (1931) 405.

11. Onsager, L. Phys. Rev. 38 (1931) 2265.

12. Coleman, B. D. and Truesdell, C. J. Chem. Phys. 33 (1960) 28.

Received December 28, 1983.

Acta Chem. Scand. A 38 (1984) No. 4 